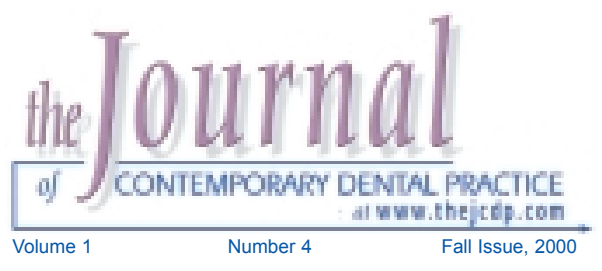

\title{
Endodontic Perforation Repair with Resin-Ionomer: A Case Report
}

\author{
Lawrence G. Breault, DMD, MS; Edward B. Fowler, DDS, MS
}

COL Patrice D. Primack, DDS, MS

\begin{abstract}
Root perforations are significant complications of endodontic treatment. However, when teeth are of strategic value, perforation repair is clearly indicated. The successful utilization and placement of a subgingival resin-ionomer restoration to repair an endodontic root perforation is demonstrated.

Sustained tissue health and minimal probing depths at the surgical site demonstrate clinical success.

Keywords: Dental restoration, resin-ionomer, subgingival, endodontic perforation, repair

Disclaimer: The opinions expressed in this article do not represent the views of the United States Department of Defense, Department of the Army, or the United States Army Dental Corps.
\end{abstract}




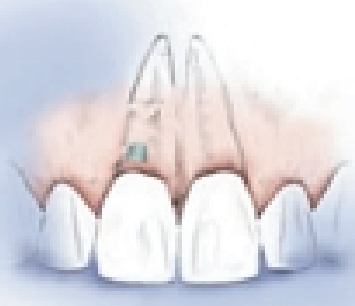

Introduction

Maintaining the integrity of the natural dentition is essential for full function and natural esthetics. ${ }^{1}$

Endodontic therapy can play a vital role in achieving this goal. Technical problems do occur occasionally during endodontic treatment, one of which is perforation of the root canal wall during mechanical debridement. This can significantly impact the long-term prognosis of the tooth. ${ }^{2}$ Perforations may be induced by iatrogenic causes, resorptive processes, or caries. ${ }^{1}$

Treatment of endodontic perforation depends on recognition of the condition, clinician training and experience, location and size of the perforation, and the timing of the therapeutic intervention. ${ }^{2,3}$ Depending upon the size and location of the perforation, repair may be achieved either through a conservative, non-surgical technique or by employing surgical intervention. In all cases, these defects must be sealed to prevent the exodus of noxious elements from within the tooth that can further irritate the associated periodontal tissues. $^{4}$

The prognosis of endodontically treated teeth with perforations is dependent on the prevention of bacterial infection of the perforation site. ${ }^{5}$ The time between perforation and repair is one of the most critical factors for success. ${ }^{1,5}$ Early treatment will significantly enhance success. The location of the perforation along the root will also greatly influence the overall tooth prognosis. When the perforations are located at the alveolar crest or coronal to it, the success rate in repairing the defect is the lowest. This is due to the fact that crestal perforations are the most susceptible to epithelial migration and rapid periodontal pocket formation. ${ }^{5,6}$ If the tooth can be salvaged, the clinician might consider other means of therapy, such as surgical crown lengthening or orthodontic extrusion in order to place the perforation supragingivally. Furthermore, a perforation that involves a furcation usually has a doubtful prognosis and extraction is often the only recourse. ${ }^{1,7}$ Perforations in the coronal third of the root which are surrounded by a healthy periodontium, in other words ones which do not communicate with the gingival sulcus, usually have a fair prognosis. ${ }^{1}$ Finally, perforations in the midroot to apical third, which do not communicate with the oral cavity, often have a good prognosis. The key to successful repair is an immediate seal of the defect. ${ }^{1,7,8}$

The size of the perforation is also critical for success. A small perforation is usually associated with less tissue destruction and inflammation. ${ }^{5,7}$ Smaller perforations also allow better control of sealing materials without extrusion into the surrounding periodontium. ${ }^{5}$ The treatment plan for perforation repair depends on the visibility and accessibility of the perforation, size of perforation, associated periodontal condition, and strategic importance of the tooth. ${ }^{1}$

Various materials have been utilized for nonsurgical perforation repair. Some of the most investigated materials include: amalgam, IRM, superEBA cement, gutta-percha, calcium hydroxide alone, and calcium hydroxide or Klorapercha N-O covered with amalgam or guttapercha. $1,2,6,9-12$ Other materials utilized have included dentin chips, hydroxyapatite, glass ionomer cements, and Plaster of Paris., ${ }^{7,1314}$ In a recent in-vitro study by Fuss and co-workers, 15 silver glass ionomer cement was found to significantly decrease leakage in furcation perforations compared to amalgam. The ultimate goal is to seal the defect with a biocompatible material and maintain an intact periodontal attachment apparatus. ${ }^{2}$

Surgical repair of endodontic perforations are usually reserved for defects that are not amenable to other treatment modalities. ${ }^{1,5}$ It is also utilized when a more conservative repair has failed, or a concomitant management of a periodontal defect is indicated. ${ }^{5}$

Recently, resin-ionomers have been successfully utilized for subgingival restorations (external root 
resorptive lesions, root fractures, and root perforations of anterior teeth). ${ }^{15-17}$ Resin-ionomer (Geristore, Den-Mat Corp, Santa Maria, CA) is a small particle, hydrophilic, nonaqueous resin combined with a photoinitiator and glass powder formulation. The advantages of the resin-ionomer are its insolubility in oral fluids, increased adhesion, high strength, and dual cure capability. Resin-ionomers also incorporate these attributes: low cure shrinkage, low thermal expansion, and extended fluoride release found in the traditional glass ionomers. ${ }^{16,17}$

Traditional clinical applications for resin-ionomers include: erosive lesions in geriatric patients, fixed prosthetics and resin bonded retainer cementation, porcelain repair, bonded amalgam restorations, core material, and pediatric restorations. ${ }^{16,17}$

Dragoo demonstrated clinically and histologically the biocompatibility of these resin-ionomer restorations. ${ }^{16}$ The formation of an epithelial and connective tissue adherence to the resin-ionomer represents a significant advancement in the ability to restore previously considered hopeless teeth. $^{16,18}$

The purpose of this report is to present a case report demonstrating the continued clinical success of resin-ionomer utilization in an anterior endodontic root perforation repair.

\section{Case Report}

A healthy 32-year old man was referred to the periodontic department for treatment of deep periodontal probing depths and radiographic bone loss associated with the maxillary right central incisor (tooth \# 8) (Figure1).

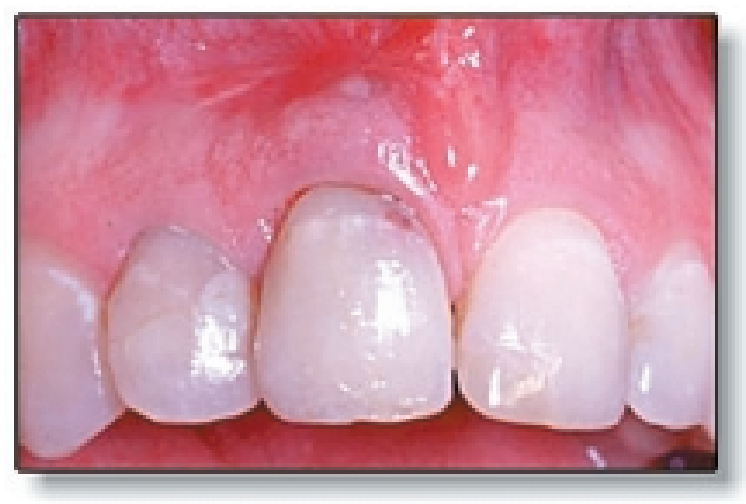

Pre-surgical presentation of maxillary right central incisor (tooth \#8)
The patient reported a history of trauma (bicycle accident) to both his right central and lateral incisor teeth approximately 20 years ago. The patient reported receiving endodontic treatment for these teeth following this trauma (Figure 2).

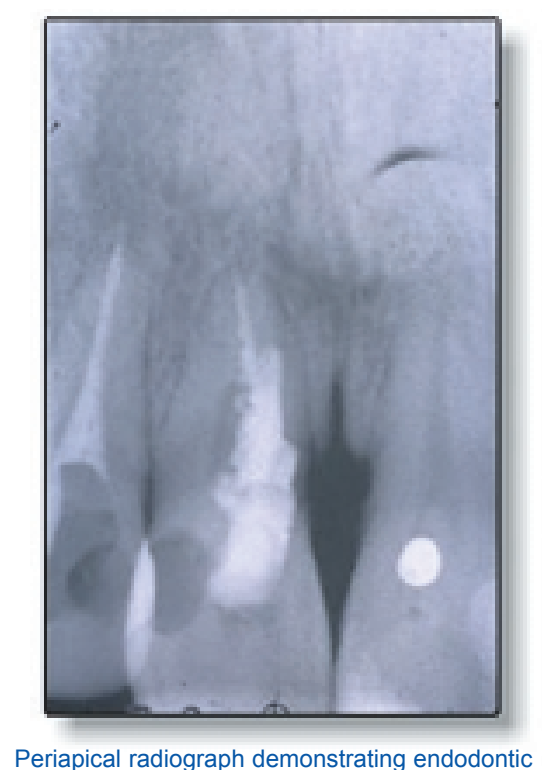
perforation and bone loss associated with \#8

Figure 2.

A diagnosis of a failed endodontic root perforation repair was made. Treatment options were discussed with the patient including extraction with subsequent restoration with either fixed prosthetics or a dental implant. The patient desired to attempt to retain this tooth, and a root perforation repair was planned with resin-ionomer.

Following a 60 second presurgical chlorhexidine rinse and administration of local anesthesia, a full thickness flap was reflected to expose the lesion (Figure 3).

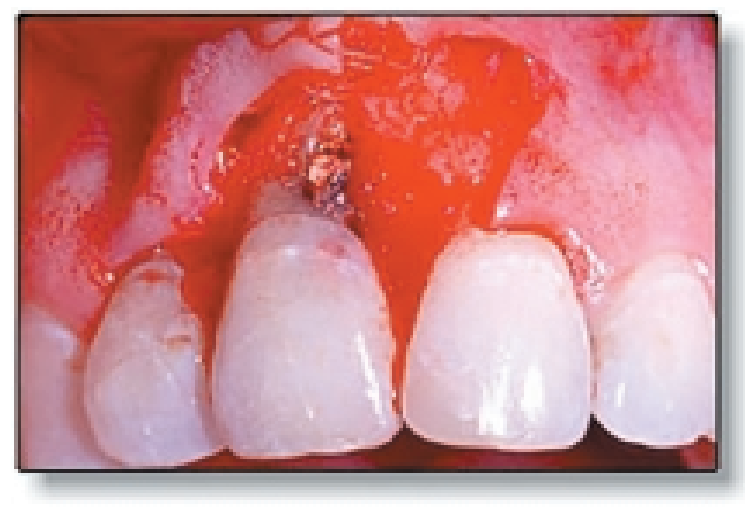

Perforation exposed following flap reflection and debridement. Note: Flap reflection should have included the distal papilla of tooth \#9.

Figure 3.

Figure 1. 
Surgical exposure and soft tissue debridement of the area revealed an extensive lesion involving the mesial-facial aspect of the root. A \#6 round carbide bur was used to remove excessive guttapercha from the perforated area as well as to expose a margin of root surface (Figure 4).

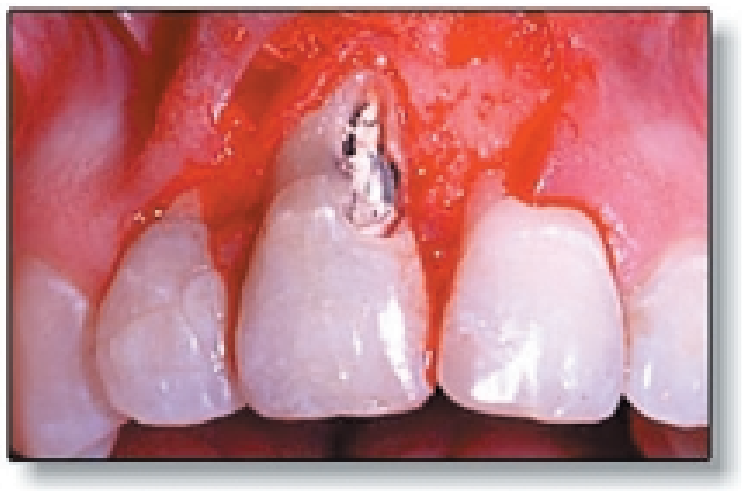

Excessive gutta-percha removed from area of root perforation.

Figure 4.

Since glass ionomer resins will bond to root structure, no definitive mechanical preparation of the tooth is mandatory, although slight mechanical retentive grooves were utilized in this case.

Following acid etching and bonding of the root surface, the restorative material was placed, light cured, and allowed to chemically cure for 10 minutes. The resin-ionomer was then contoured with a flame shaped finishing bur (Figure 5).

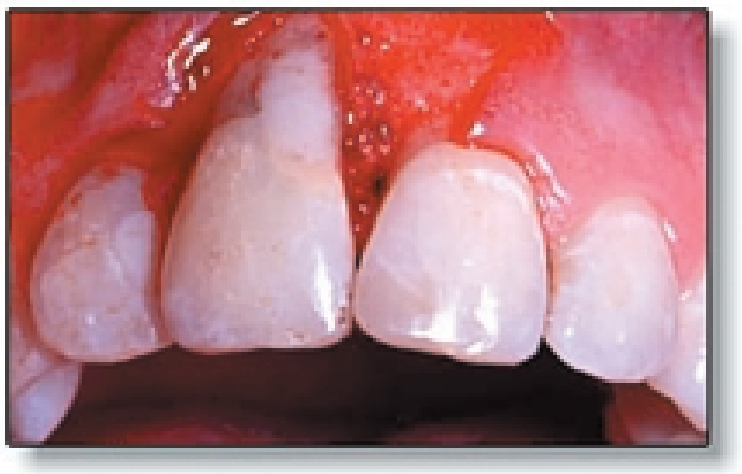

Resin-ionomer placed and contoured with a finishing bur

Figure 5.

After copious sterile saline irrigation of the surgical site, the flap was replaced and sutured with 4-0 black silk (Figure 6).

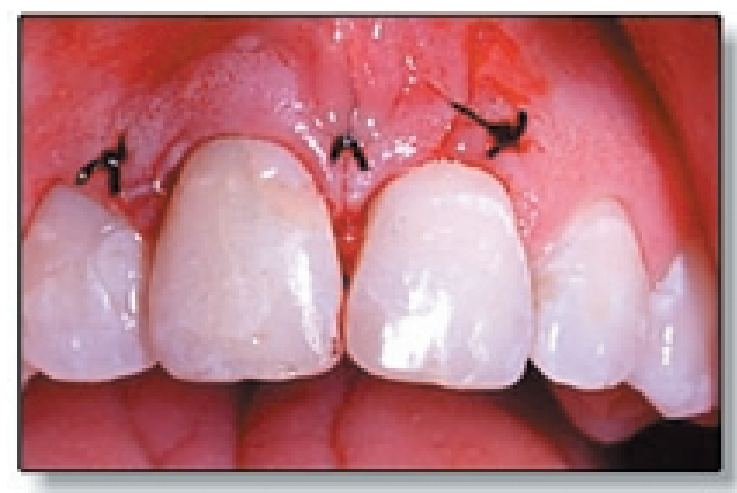

Flap sutured with 4.0 black silk sutures.

Figure 6.

Healing was uneventful. Other than soreness in the area, the patient voiced no complaints.

Sutures were removed one week after surgery. Tissues were judged to be of good tone and color, although a slight gingival dehiscence was noted on the facial of the left central incisor. This dehiscence could have probably been avoided by including the distal papilla of the left central incisor in the initial flap elevation (Figure 7).

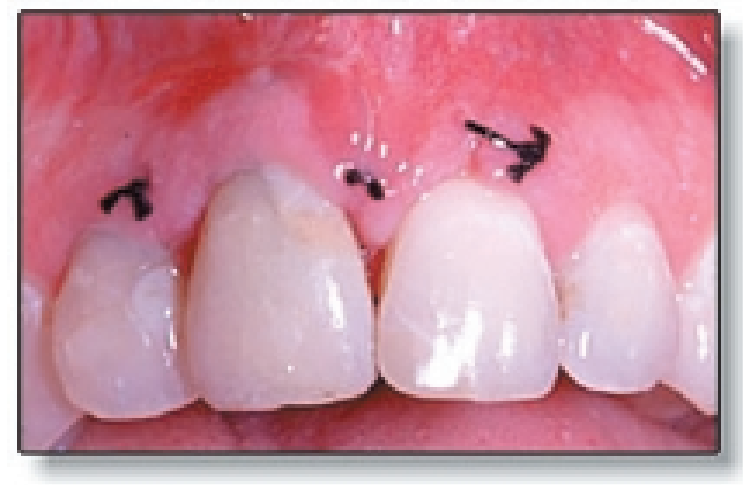

One-week surgical follow-up. Good tissue tone and color, however, a slight dehiscence has developed on the mid-facial of tooth \#9.

Figure 7.

At 7-months post surgery, the patient was clinically healed. Periodontal probing depths were measured to be less than $3 \mathrm{~mm}$ (Figure 8). 


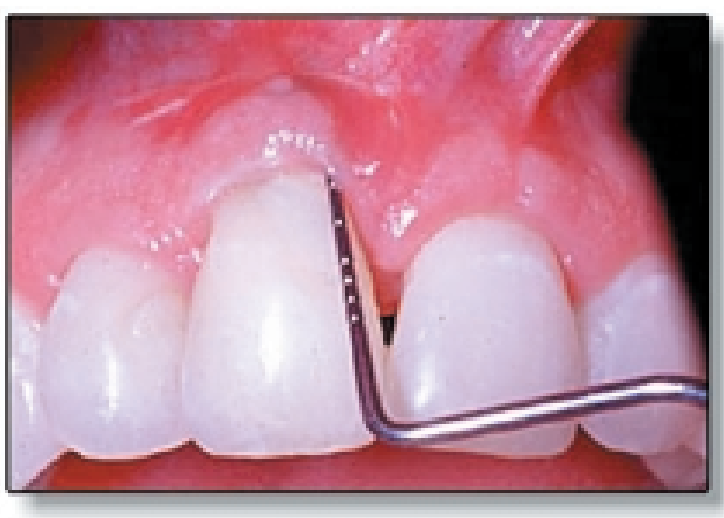

Seven-month post surgical follow-up reveals good tissuehealing with less than $3 \mathrm{~mm}$ probing depth and excellent tissue response to the resin-ionomer.

\section{Figure 8.}

In addition, the anterior esthetics were maintained with excellent tissue response to the resinionomer.

\section{Discussion}

Wilson and Kent first introduced glass ionomer restorative materials in 1972. ${ }^{19}$ The popularity of these materials for use in crown cementations, cavity liners, core build-up material, and as independent restorations has dramatically increased over the past two decades..$^{20,21}$ Dragoo's clinical and histological investigation of resinionomer demonstrates a biocompatibility to both soft and hard tissues. ${ }^{16}$ These results present an opportunity for alternative techniques in certain iatrogenic and traumatic subgingival lesions. As an additional benefit, fluoride release from resinionomer may positively affect bacterial plaque biochemistry through an alteration of carbohydrate metabolism. ${ }^{17-19}$ This release may result in a decrease in localized gingival inflammation independent of patient home care. ${ }^{16}$

\section{Conclusion}

Resin-ionomer, as a relatively new dental restorative material, has been successfully utilized for treatments of tooth abfractions, external root resorption, and root perforation repair. ${ }^{16-18}$ The present case report is an additional demonstration of its efficacy. With its enhanced biologic compatibility, resin-ionomer restorations should be incorporated into the clinician's armamentarium for the treatment of endodontic root perforations. This single case report demonstrates a resin-ionomer technique that can be successfully utilized in the surgical management of root perforations. 


\section{References}

1. Alhadainy HA. Root Perforations: A review of literature. Oral Surg Oral Med Oral Pathol1994;78: 368-374.

2. Duggins LD, Clay JR, Himmel VT, Dean JW. A combined endodontic retrofill and periodontal guided tissue regeneration technique for the repair of molar endodontic furcation perforations: Report of a case. Quintessence Int 1994;25: 109-114.

3. Seltzer S, Sanai I, August D. Periodontal effects of root perforations before and during endodontic procedures. J Dent Res 1970;49: 332-339.

4. Delivanis PD, Goerig AC. Repair of Perforations. Quintessence Int 1981;12: 985-992.

5. Fuss Z, Trope M. Root perforations: Classification and treatment choices based on prognostic factors. Endod Dent Traumatol 1996;12: 255-264.

6. Petersson K, Hasselgren G, Tronstad L. Endodontic treatment of experimental root perforations in dog teeth. Endod Dent Traumatol 1985;1: 22-28.

7. Himel VT, Brady J, Weir J. Evaluation of repair of mechanical perforations of the pulp chamber floor using biodegradable tricalcium phosphate or calcium hydroxide. J Endod 1985;11: 161-163.

8. Martin LR, Gilbert B, Dickerson AW. Management of endodontic perforations. Oral Surg Oral Med Oral Pathol 1982;54: 668-677.

9. Mannocci F, Vichi A, Ferrari M. Sealing ability of several restorative materials used for repair of lateral root perforations. J Endod 1997;23(10): 639-641.

10. Bogaerts P. Treatment of root perforations with calcium hydroxide and SuperEBA cement: A clinical report. Int Endod J 1997;30: 210-219.

11. El Deeb ME, El Deeb M, Bavivi A, Jensen JR. An evaluation of the use of amalgam, cavit and calcium hydroxide in the repair of furcation perforations. J Endod 1982;8: 459-466.

12. Cathey GM. Molar endodontics. Dent Clin North Am 1974;18: 345-366.

13. Lemon RR. Nonsurgical repair of perforation defects: Internal matrix concept. Dent Clin North Am 1992;36: 439-457.

14. Alhadainy HA, Himel VT. An in vitro evaluation of plaster of Paris barriers used under amalgam and glass ionomer to repair furcation perforations. J Endod 1994;20: 449-452.

15. Fuss Z, Abramovitz I, Metzger Z. Sealing furcation perforations with silver glass ionomer cement: An in-vitro evaluation. J Endod 2000;26: 466-468.

16. Dragoo MR. Resin-ionomer and hybrid-ionomer cements: Part II. Human clinical and histologic wound healing responses in specific periodontal lesions. Int J Periodontics Restorative Dent 1997; $17:$ 75-87.

17. Scherer W, Dragoo MR. New subgingival restorative procedures with Geristore resin ionomer. Pract Periodontics Aesthet Dent 1995 Jan-Feb;7(1 Suppl): 1-4.

18. Shuman IE. Repair of a root perforation with a resin-ionomer using an intentional replantation technique. Gen Dent 1999;47: 392-395.

19. Wilson AD, Kent BE. A new translucent cement for dentistry. Br Dent J 1972;132: 133-135.

20. Titley KC, Smith DC, Chernecky R. SEM observations of the reactions of the components of a light activated glass polyalkenoate (ionomer) cement on bovine dentin. J Dent 1996;24(6): 411-416.

21. Swift EJ, Schulein TM, Reinhardt JW. Use of glass-ionomer cements by lowa dentists. Gen Dent 1990;38(6): 450-453.

\section{Acknowledgment}

The authors would like to thank Mrs. Dayna Breault and Mrs. Michelle Fowler for assistance with manuscript preparation. The authors would also like to thank Mrs. Lisa Bell, Dental Assistant, for help in photo documentation. 


\section{About the Authors}

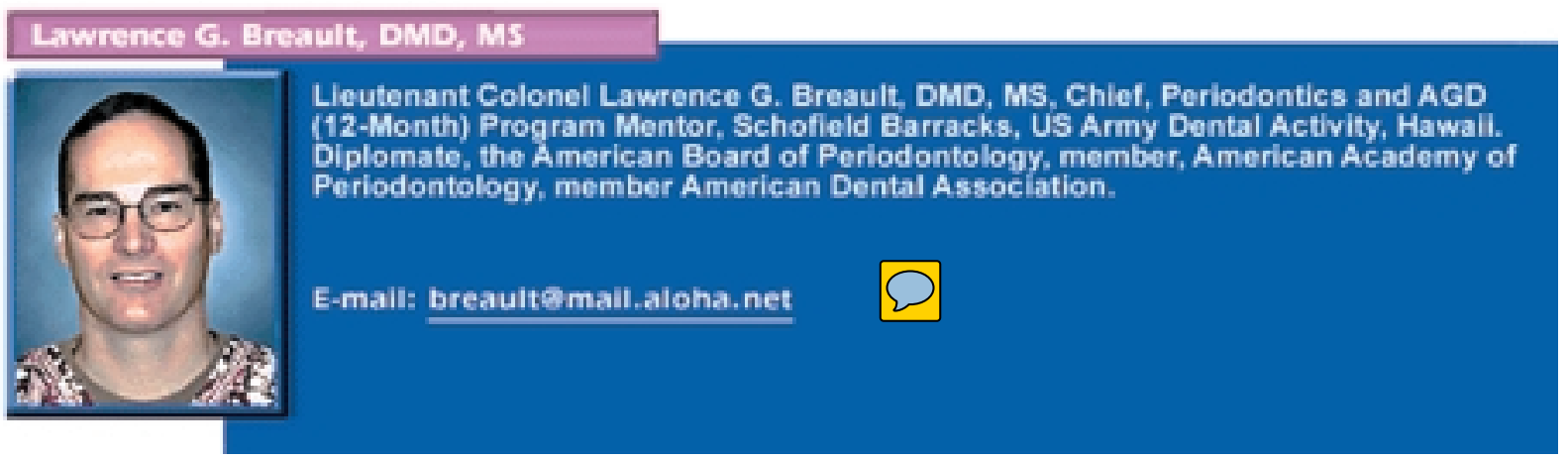

\section{Edward B. Fowler, DDS, MS}

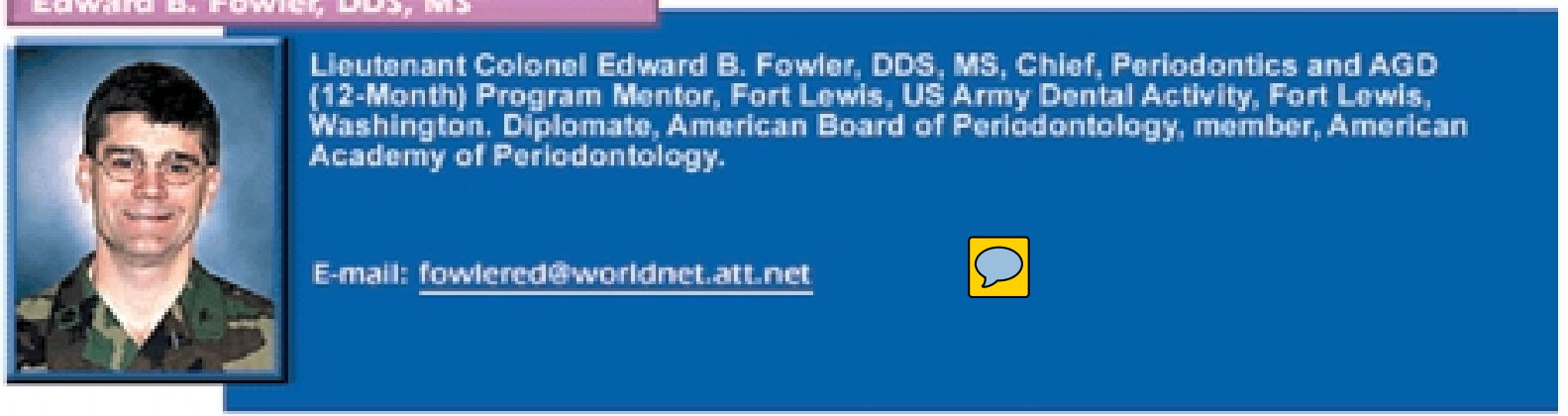

\section{Patrice D. Primack, DDS, MS}

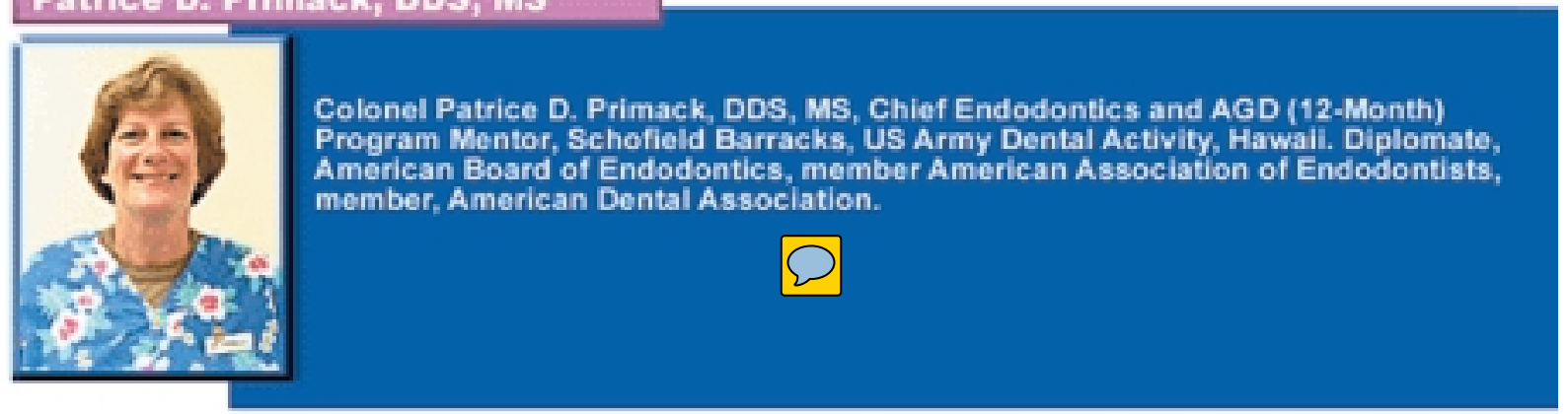

